\title{
HVet: um Modelo de Hipervídeo Aplicado ao Ensino de Cirurgia Veterinária $^{1}$
}

\author{
Claudio Afonso Baron Tiellet - UFSM - tielletcab@yahoo.com.br \\ José Valdeni Lima - UFRGS - valdeni@inf.ufrgs.br \\ Eliseo Berni Reategui - UFRGS - eliseoreategui@ gmail.com
}

\begin{abstract}
Resumo
Este trabalho propõe um novo modelo de navegação em hipervídeo com o objetivo de torná-lo ainda mais eficaz como ferramenta educacional. Nos modelos de hipervídeo hoje existentes, os links são unidirecionais, possibilitado ao usuário explorar um tópico de cada vez para cada objeto/link clicado. No modelo proposto, os links são multidirecionais, permitindo explorar informações relativas a diversas facetas de um mesmo objeto/link. Este modelo baseia-se no trabalho de co-links de Alex Primo, os quais também permitem uma navegação multi-direcional em hipertextos. O modelo de hipervídeo proposto busca adequação da carga cognitiva para uso no ensino de cirurgia, e foi empregado na construção de um sistema para armazenamento e apresentação de hipervídeos. O artigo apresenta este sistema, bem como uma discussão sobre seu uso na Educação.
\end{abstract}

Palavras-chaves

hipervídeo, hipermídia, anotações, co-links, aprendizagem.

\section{HVet: a Hypervideo Model Applied to the Teaching of Veterinary Surgery}

\begin{abstract}
This work proposes a new navigation model for hypervideos with the main goal of making them more efficient as an educational tool. In the current hypervideo models, links have one single destination, enabling users to explore one topic at a time for each object/link selected. In the model proposed here, links may have several destinations, enabling users to explore information related to different aspects of the same object/link. This model is based on Alex Primo's co-link work, which proposes multidirectional navigation in hypertexts. The model presented here tries to adjust hypervideo cognitive load for the use in surgery teaching, and it has been employed in the development of a system for the storage and presentation of hypervideos. This paper presents the hypervideo system, as well as a discussion about its use in Education.
\end{abstract}

\section{Keywords}

hypervideo, hypermedia, annotations, co-links, learning.

\footnotetext{
${ }^{1}$ Este trabalho é um resumo da proposta de tese do aluno Claudio Afonso Baron Tiellet - PPGIE. 


\section{Introdução}

Muitos autores já comprovaram que o vídeo é uma tecnologia muito eficaz na Educação, como (Moram, 1995; Afonso, 2004). No entanto, por não ser um recurso interativo, o vídeo muitas vezes não permite explorar de maneira satisfatória os conteúdos presentes. Surge então o Hipervídeo (HV) como uma proposta que estende as funcionalidades do vídeo. Mesmo sendo uma tecnologia recente, esta já é considerada por muitos o futuro das tecnologias de informação em rede, pois sua estrutura baseada em linguagem videográfica tem conceito e lógica semelhante ao hipertexto, proporcionando inúmeras relações dialógicas entre autor e usuário (Chambel e Guimarães, 2000). Segundo os mesmos autores, a utilização intensiva de vídeo, a mobilidade e a reutilização de conteúdos existentes está transformando a forma como consumimos e produzimos materiais multimídia.

Apesar de ter um uso ainda incipiente, o hipervídeo já pode ser encontrado em algumas aplicações na área de publicidade, nas quais os profissionais envolvidos exploram a novidade da interação para aumentar o impacto da apresentação de produtos/serviços. $\mathrm{Na}$ Educação, a utilização do hipervídeo também é recente, tendo como principal objetivo permitir que os estudantes explorem mais profundamente os conteúdos apresentados nos vídeos. Quando aplicado à Educação, no entanto, percebe-se que uma deficiência ocorre quando um estudante deseja saber mais sobre um detalhe que pode ter múltiplas facetas no hipervídeo. Nos modelos de hipervídeo existentes, ao clicar sobre um objeto do vídeo uma única descrição ou explicação é apresentada, negligenciando o fato de que muitas perguntas podem ser feitas sobre o objeto selecionado.

Este artigo propõe uma solução para este problema através da utilização de co-links, uma técnica que permite a inserção de várias opções de navegação em um único link, possibilitando também a colaboração dos usuários na construção destes (Primo, 2004). Aqui, os co-links são usados de maneira análoga, permitindo explorar de forma mais objetiva, múltiplas opções escondidas atrás do que seria um único link. Esta oferta de possibilidades de navegação distintas leva o aprendiz a refletir mais profundamente sobre o que sabe, e o que realmente está buscando ao selecionar determinado link. Esta ação deliberada na busca por determinado tipo de conhecimento promove um tipo de aprendizagem que pode ser classificado por como aprendizagem significativa (Ausubel, 1982; Moreira 1998). Segundo Ausubel, são três os requisitos para que esta ocorra. Primeiramente, o aprendiz deve ser confrontado com um conhecimento novo, apresentado de maneira lógica. Em segundo lugar, este aprendiz já deve possuir uma rede de informações e conceitos em sua estrutura cognitiva que lhe permitam conectar o novo conhecimento aos que já existem; Em terceiro lugar, o aprendiz deve tomar deliberadamente a atitude de aprender, buscando associações entre o conhecimento novo e antigo. Deste modo, ao disponibilizarmos aos estudantes múltiplos links em um 
vídeo, tornando-o interativo, permitimos que os aprendizes tomem uma postura ativa na busca por conhecimentos que, em determinado instante, lhe pareçam mais apropriados para serem conectados a suas redes de conhecimento.

A próxima seção apresenta o tópico vídeo, enfatizando o impacto que este teve na Educação. Na seção 3, o hipervídeo é apresentando, mostrando trabalhos importantes e que são referência na área. Na seção 4 um novo modelo de hipervídeo é proposto, nos quais foram incorporados co-links para enriquecer a estrutura de navegação e aumentar ainda mais a capacidade de interação dos estudantes com os hipervídeos. Na seção 5 são levantadas e discutidas questões pedagógicas relacionadas à aplicação do hipervídeo na educação, e na última seção são apresentadas conclusões e propostas para trabalhos futuros.

\section{O Vídeo}

$\mathrm{O}$ vídeo, elemento central do $\mathrm{HV}$, carrega consigo características de recurso pedagógico, desenvolvendo atitudes perceptivas múltiplas nos estudantes. O vídeo nos traz uma significação apoiada no discurso verbal-escrito, partindo do concreto, do visível, do imediato (Moram, 1995). Na aprendizagem é freqüentemente destacado por suas funções lúdica, motivadora, informativa, avaliativa, conceitual, documental, metalingüística e procedimental (Correia et al, 2004). Traz embutidas características peculiares demonstrando sua potencialidade no ensino/aprendizado em inúmeras áreas, principalmente quando não se pode dispor do objeto real de estudo, como demonstrado em vários trabalhos, sobre a influência da imagem na percepção.

O uso de vídeo na educação, até pouco tempo, teve o poder computacional como barreira, mudando de forma entusiástica a partir da disseminação das novas tecnologias computacionais. Graças à largura de banda, hoje é possível assistir apresentações em tempo real, apresentações síncronas e com boa qualidade. A realidade pode ser mostrada de forma dinâmica, e o usuário determina local e tempo que melhor lhe convém, favorecendo os novos paradigmas do aprendizado. A partir do momento em que se torna digital, o acesso, transmissão e armazenamento do vídeo se tornam também mais fáceis. Assim, "as novas tendências no sentido da convergência e integração das mídias estão a transformar o vídeo num meio dominante" (Gibbs e Davis, 1992).

\section{O Hipervídeo}

Gradualmente, a Internet se transformou numa fonte "imagética" de conhecimento e informações, explorando o vídeo de maneira intensiva. Inúmeros sítios têm-se destacado estatisticamente como os mais visitados, sendo seus conteúdos freqüentemente baseados no vídeo. Logo, buscam-se alternativas com vistas ao uso de ferramentas que tenham como elemento central o poder da imagem. $\mathrm{O}$ hipervídeo, com suas origens no hipertexto de Ted Nelson (1974), é justamente uma destas tecnologias que têm no vídeo, e conseqüentemente na imagem, um de seus principais eixos. 
Usando conexões espaço-temporais, o hipervídeo permite delimitar uma área no vídeo fonte por um intervalo de tempo determinado, conectando-a a outros vídeos ou a outros conteúdos disponíveis na rede. Cabe destacar a interação proporcionada por esta técnica, que permite ao usuário sair de um papel mais passivo e tornar-se ator, capaz de definir a todo instante qual o próximo conteúdo que lhe interessa explorar.

Um dos trabalhos mais conhecidos na área de hipervídeo é o HyperCafe (Sawhney et al., 1996), no qual é possível interagir dinamicamente com um vídeo para seguir diferentes narrativas de conversas. Apesar de permitir a navegação de um vídeo a outro, Hypercafe era sobretudo um sistema de apresentação, não permitindo a criação dinâmica de links nos vídeos (Stotts et al., 2002).

Outro projeto bastante conhecido é o editor de hipervídeo Hyper-Hitchcock, que permite criar links entre os vídeos e visualizar detalhes de um vídeo quando solicitado (detail-on-demand) (Shipman e Wilcox, 2005). Os autores do trabalho argumentam que este tipo de abordagem é adequada para vídeos que explicam "como fazer", nos quais um tópico explicado num nível mais abstrato pode ser detalhado quando o usuário clica em um elemento para visualizar aspectos mais específicos. Contudo, apesar dos avanços apresentados pela ferramenta, a partir de um conjunto de experimentos alguns problemas foram identificados no editor. Por exemplo, dificuldades na conexão dos segmentos de vídeo e retorno ao vídeo original (Shipman et al., 2005).

Outro problema identificado na área, mesmo anterior aos projetos acima citados, é a dificuldade de mapeamento e seleção das regiões desejadas para serem linkadas, quer de forma manual ou automática (Dakss, 1997). Algumas soluções comerciais já definiram possibilidades bastante intuitivas para o problema, como é o caso da ferramenta VideoClix. Nela, um vídeo é linkado a outros vídeos ou a páginas web através de uma interface gráfica. Um sistema de auto-tracking permite ainda rastrear e acompanhar dinamicamente um objeto demarcado no vídeo pelo usuário. Em outra ferramenta, o Asterpix, além do usuário poder realizar seu trabalho online e definir áreas clicáveis através de uma interface intuitiva, o hipervídeo resultante é automaticamente disponibilizado na web.

Apesar do progresso tanto na pesquisa em hipervídeo quanto no desenvolvimento de ferramentas comerciais, nenhum dos modelos de hipervídeo existentes trata de um problema já existente em hipertextos e ainda mais acentuado em hipervídeos: quando o usuário seleciona um determinado link (uma palavra ou frase em um hipertexto, um objeto em uma cena em um hipervídeo) várias possibilidades poderiam estar associadas ao link selecionado. Nos hipertextos, Alex Primo (2007) propõe a utilização de co-links, os quais oferecem múltiplas opções de navegação para um mesmo link. Empregados com sucesso em algumas implementações ao nível de pesquisa, os co-links se mostraram muito úteis na expansão das possibilidades de navegação já introduzidas em narrativas hipertextuais. Neste projeto, propomos a utilização da mesma abordagem nos hipervídeos, permitindo que, ao selecionar determinado elemento de um hipervídeo, 
várias possibilidades de navegação sejam apresentadas ao usuário. A seção XXX descreve esta abordagem num maior nível de detalhe.

\subsection{Anotações}

O emprego de anotações na web cresceu imensamente nos últimos anos, permitindo que os usuários da rede se expressassem com relação a itens dos mais diversos, dentre estes, postagens de outros usuários. As anotações são vistas como caminhos-chave que permitem que os hipertextos cresçam e aumentem de valor (Marshall, 1998). Fator positivo é que o usuário passa de leitor a autor, aumentando seu interesse subseqüente pelo resultado das ações. Assim como no papel, representam observações que os usuários criam para si, ou para outros, referindo-se a uma parte específica do conteúdo, como demarcar uma imagem ou área, ressaltar palavras ou parágrafos. Em alguns casos, como neste trabalho, as anotações se tornam mecanismos poderosos por possuírem um contexto definido.

As anotações são categorizadas em (Petkovic et al, 2005): a) Criação de link: o leitor procura clarificar as conexões entre a informação contida no documento, com outra informação, para si, ou para a comunidade; b) Construção de caminhos: usuário(s) expert(s) procura $(\mathrm{m})$ construir mapas ilustrativos onde existe muita informação, ou complexas, para auxiliar no entendimento; c) Comentário: o usuário relata fatos ou manifesta opinião; d) Destaque ou marcação: a finalidade é simplesmente chamar a atenção para um determinado segmento; e) Decentralização de autoria: o anotador procura demonstrar seu próprio conhecimento em um determinado tópico em particular; f) Registros de leitura: o usuário a usa para auxiliar na memorização e marcação de documentos, e o que foi considerado importante na leitura; g) Memória comunitária: as anotações servem de veículo para exprimir a evolução do pensamento ou de um arquivo resultante da contribuição da comunidade.

No hipervídeo, anotações têm sido empregadas na visualização ativa, permitindo a criação e edição de anotações, a visualização de vídeos anotados, a importação de anotações de autores, bem como o compartilhamento de documentos de um determinado programa de vídeo (Correia, 2004).

\section{O Modelo HVet}

[...] o ambiente hipervídeo tem que ser concebido de modo a envolver o utilizador de uma forma que seja levado a "parar, pensar, e relacionar" diferentes tipos de informação" (Chambel, T. e Nuno, G,.2000).

Os modelos de vídeo existentes permitem criar conexões através de links unidirecionais. A idéia deste trabalho é incorporar a tecnologia de co-links aos hipervídeos, dotando-os da possibilidade de inclusão de links para múltiplas direções. 
A tecnologia Co-link, concebida por Alex Primo (2004), tem como um de seus objetivos permitir a construção social do conhecimento, buscando a colaboração dos usuários. Ao fazer isso, o link (um conteúdo) deixa de ser propriedade do autor e passa a gerar uma força centrípeta, atraindo a inclusão coletiva de novos caminhos.

Partindo deste mesmo princípio, os co-links foram associados ao hipervídeo, aumentando seu grau de interatividade e possibilitando a participação de diversos usuários na construção dos links.

No modelo aqui proposto o co-link vem a auxiliar numa navegação mais precisa, apontando para o que realmente o usuário busca. Como exemplo, podemos citar a frustração que o usuário pode sentir ao clicar em determinado link e não encontrar o esperado. Para minimizar este problema, podemos oferecer diversas opções dentro da concepção de um tema (Bianchini, 2000). O número de opções estabelecido ao se fazer a concepção do conteúdo é ilimitado, havendo ainda a possibilidade do próprio usuário criar links. Neste sentido, o usuário passa da passividade a co-autoria.

\subsection{A ferramenta HVet}

A partir deste modelo, foi construído um repositório para hipervídeos utilizando as linguagens de programação $\mathrm{PhP/Javascript,} \mathrm{e} \mathrm{HTML.} \mathrm{Este} \mathrm{repositório} \mathrm{tem} \mathrm{o} \mathrm{fim}$ específico de armazenar hipervídeos de cirurgia, constantes da grade curricular da disciplina de Princípios de Técnica Cirúrgica Veterinária. A Figura 1 ilustra a página inicial do repositório.

$\mathrm{Na}$ área $\mathrm{A}$ da figura, vemos a barra de título, o menu de interação e a caixa de busca. $\mathrm{Na}$ região $\mathrm{B}$, vemos oito imagens minimizadas representando cada uma um hipervídeo de uma cirurgia, acompanhadas de um título e de um autor. $\mathrm{Na}$ área $\mathrm{C}$ temos uma listagem dos vídeos mais consultados. Na área $\mathrm{D}$ encontramos o texto de apresentação da página.

Ao selecionar um item, tem-se acesso à página do hipervídeo propriamente dita. Nesta página foi utilizada a ferramenta $\operatorname{VideoClix}^{2}$ para disponibilizar os hipervídeos e permitir aos usuários interagir com eles. Seguindo disposição sugerida pelo programa, optou-se pelo uso de quatro frames (Figura 2): frame (A) - barra de títulos, sistema interno de busca; (B) - área permanente do hipervídeo; (C) - área interna destino da maioria das mídias usadas; (D) - barra com opções de links interativos, anotação, compartilhamento de conteúdo através de e-mail, e demais conexões permanentes.

Sempre que um objeto for clicado no hipervídeo, à direita do mesmo (E, Figura 2), e na forma de menu suspenso (рорир), são ofertadas opções de link a novas mídias. No referido menu são introduzidas, caso as mídias destino possuírem imagem, miniaturas na forma de thumbnails. Um texto introdutório sempre é apresentado (C), permitindo sua re-edição tanto de forma individual quanto de forma colaborativa.

\footnotetext{
${ }^{2}$ Videoclix ${ }^{\circledR}$ - Programa cedido pela empresa VideoClix Technologies Inc., para uso na tese do autor. 


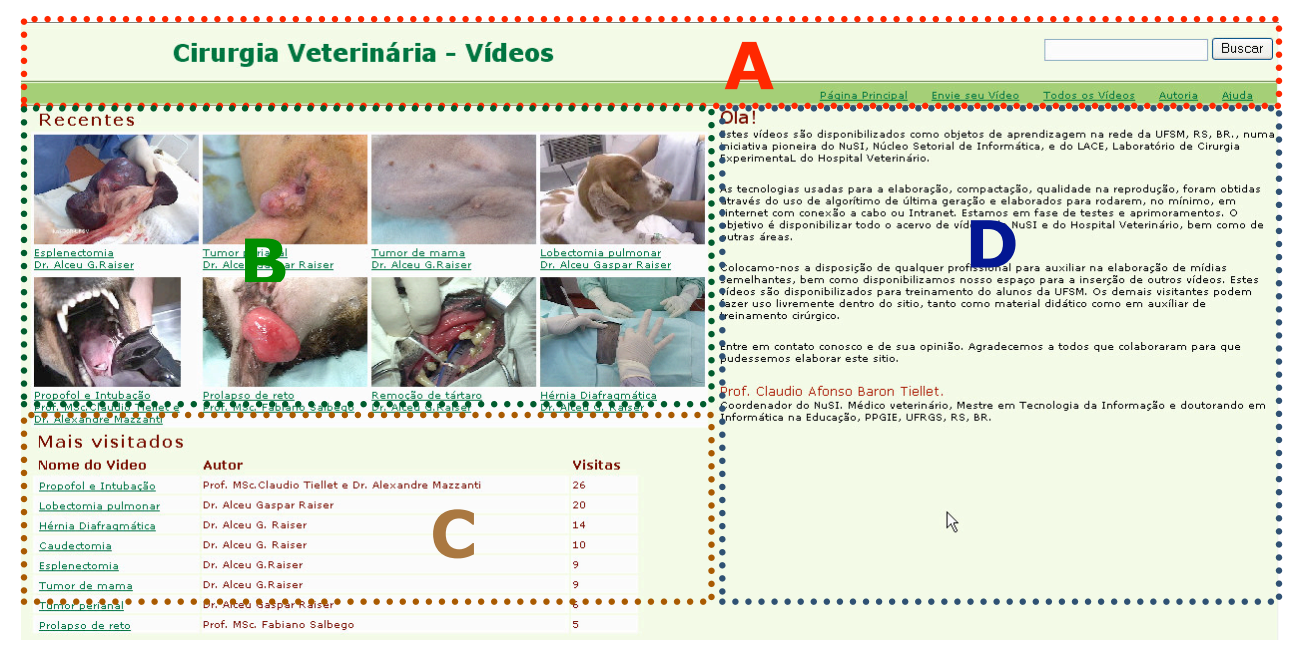

Figura 1- Página inicial do repositório de vídeos sobre cirurgia

A prototipação deste modelo, para aplicação específica no ensino de cirurgia, segue a proposição apresentada para projeto e desenvolvimento de ambientes educativos por Falkembach (2005).
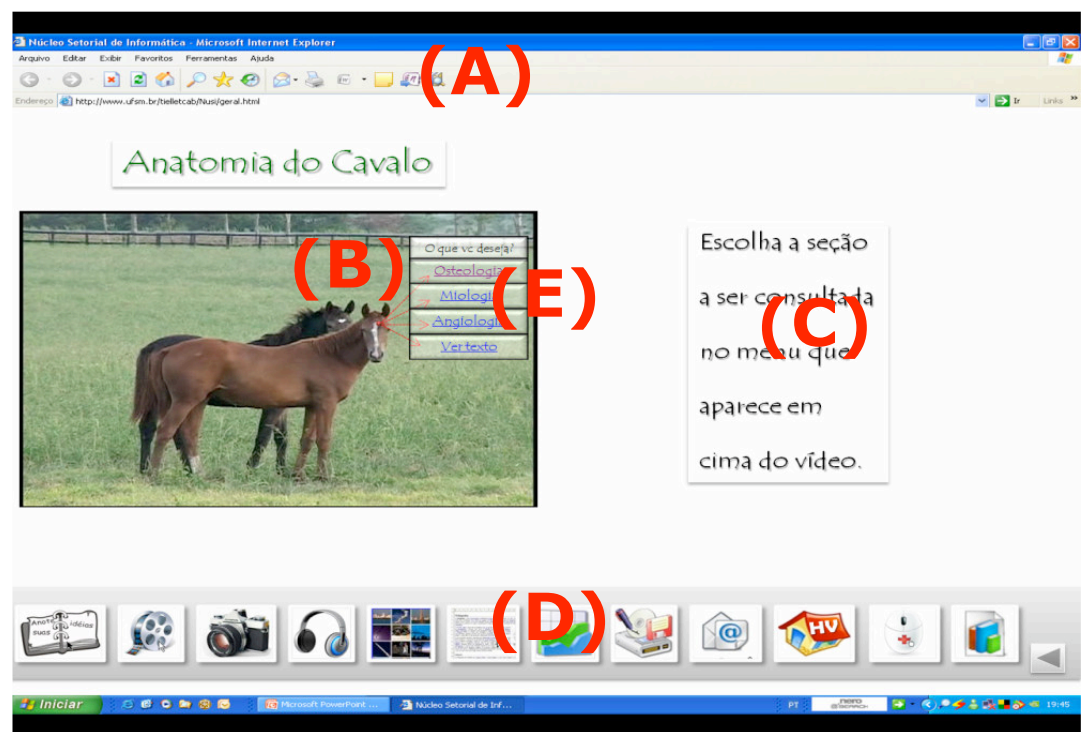

Figura 2 - Página com hipervídeo escolhido pelo usuário, com opções de link dentro do contexto. 


\section{Discussão}

Várias estratégias pedagógicas são usadas com estudantes que necessitam desenvolver habilidades manuais, como em cirurgia. Há que se salientar aqui que, o estudante foco deste estudo, já possui conhecimentos prévios de técnicas cirúrgicas, tanto teóricos como práticos (Pimentel, 1997). No ato prático de treinamento, são envolvidos os passos característicos de uma cirurgia, e tais procedimentos possuem envolvimento multisensorial pleno, proporcionando aprendizado eficaz.

O ensino, aprendizagem e procedimentos cirúrgicos que não são realizados durante a graduação, são um grande desafio tanto para professores como para os futuros profissionais. Uma das soluções para o problema é o enriquecimento dos conteúdos com técnicas que permitam ao futuro profissional o entendimento de como realizá-las, metodologias que lhes proporcionem e permitam a exata percepção e o procedimento diferencial com as habilidades já existentes. Saliente-se aqui que os alunos já possuem o conhecimento básico, os subsunsores fundamentais e procedimentais comuns a todo ato cirúrgico. Tal observação vai de encontro à Teoria Significativa de Aprendizagem, centrada na Psicologia Cognitiva de Ausubel (1980), pois "novas idéias e informações são aprendidas na medida em que existam pontos de ancoragem", ou seja, o conhecimento prévio faz ponte à nova informação (Tarouco, 2006 ; Novak, 1976, 1977 ; Moreira, 1977).

Em outro momento, existem inúmeros procedimentos cirúrgicos extracurriculares que fazem parte do dia a dia do profissional diplomado na área. Nos hospitais veterinários das instituições de ensino superior é normal o pronto atendimento, no qual são realizadas inúmeras cirurgias diariamente e que, sendo documentadas, proporcionam uma rica casuística não constante nos currículos. Aliados aos conhecimentos prévios do aprendiz, à experiência pessoal e ao material pedagógico disponibilizado, os hipervídeos podem permitir que o estudante atinja níveis satisfatórios de aprendizagem.

Outro aspecto abordado neste estudo e inerente à navegação em HV é uso de hiperlinks com determinada percepção do destino. Ao ser acessada a nova mídia, nem sempre o conteúdo condiz com o esperado pelo usuário, causando-lhe muitas vezes frustração e perda de tempo, fator altamente negativo em termos de aprendizado. Com vistas a contribuir na solução do problema, concebeu-se um modelo de hipervídeo no qual são empregados Co-links (op.cit.). Neste modelo, os objetos com múltiplas possibilidades de link(s) permitem que o usuário escolha um conteúdo destino, dentro de um contexto pertinente (Figura 2). No exemplo citado, em um HV de anatomia, apenas cinco opções são possíveis, conforme apresentação curricular na disciplina. Cada uma delas é representada por uma possibilidade de link, havendo, portanto, uma menor possibilidade de erro na escolha.

Elaborou-se então uma estrutura para armazenamento e apresentação de hipervídeos com base na integração de mídias, propondo uma nova estrutura para um repositório de 
vídeos elaborados em projeto hipermídia anterior ${ }^{3}$, com casuística armazenada ao longo de atividades iniciadas em 1990 (Tiellet, 2005).

\section{Conclusão}

$\mathrm{Na}$ busca de soluções que vão ao encontro de novos paradigmas propostos ao ensino, acredita-se que o HV traga novo alento, proporcionando bons resultados. Dentro do que se espera das novas tecnologias, usar $\mathrm{HV}$ é tratar do novo, do imagético, da interatividade. Os educadores concordam que, quando um estudante começa a aprender e gosta da atividade realizada, metade do seu problema está resolvido (Disney, 1994 apud Chaves, 1985).

Recentemente, Stahl et al. (2007) apresentaram trabalho onde propunham um conceito de curso com base na construção colaborativa do HV: (a) desenvolvimento da tecnologia HV; (b) pesquisa em aprendizagem com sistemas HV; (c) aplicação de pesquisa em aprendizado através da escrita de textos ou hipertextos para HV. A proposta dos autores era demonstrar como a construção colaborativa pode apoiar a construção do conhecimento em cursos superiores de psicologia. $\mathrm{O}$ curso mostrou ser próspero e bem apreciado pelos estudantes.

Assim como as anotações trouxeram maior dinamismo e interatividade ao $\mathrm{HV}$, acreditase que o uso do Co-links também possa trazer resultados significativos na Educação. A experiência com o uso de co-links em hipertextos mostra que esta é uma solução promissora para os problemas de navegação aqui relatados. Um dos próximos passos deste trabalho será testar esta combinação de tecnologias junto a alunos da Medicina Veterinária de diversas instituições no Rio Grande do Sul, dentre elas: PUC (Uruguaiana), Rural de Alegrete, Universidade Federal de Santa Maria (UFSM) e Universidade Federal do Rio Grande do Sul (UFRGS). Os resultados serão avaliados após o uso do HV durante um semestre letivo, através de testes de conhecimento e aplicações estatísticas.

A literatura tem mostrado crescentes pesquisas com o HV, como aposta no aprendizado via rede, bem como TV Digital e grandes perspectivas para EAD. Concomitante à construção de novos programas que envolvem estas tecnologias e formas da apresentação do saber científico, trata-se aqui também da formação de equipes multidisciplinares, de novos profissionais com fluência nessas tecnologias, originando novos pólos de disseminação e uso adequado dessas inovações.

\section{Referências}

AFONSO, Rui Walter Moreira Pires. Análise da integração de múltiplos formatos no software educativo multimedia. Tese de doutorado. Braga, 2004. Portugal.

\footnotetext{
${ }^{3}$ NuSI, Centro de Ciências Rurais da Universidade Federal de Santa Maria, RS, Brasil. 
AUSUBEL, D.P. A aprendizagem significativa: a teoria de David Ausubel. São Paulo: Moraes, 1982.

BIANCHINI, Adelaide. Modelo referencial de hipermedio, basado en teoría de grafos, para minimizar el problema de desorientación del usuário. Publicado en las Memorias del Congreso Internacional de Computación - CIC 2000, pp.121-130. México, Noviembre 2000.

CHAMBEL, T.; GUIMARÃES, N. Aprender com Vídeo em Hipermédia. 2000. Departamento de Informática. Faculdade de Ciências da Universidade de Lisboa Edifício C5, Piso1, Campo Grande, 1749-016 Lisboa.

CHAMBEL, Teresa, ZAHN, Carmen,; FINKE, Matthias. "Hypervideo and Cognition: Designing Video-Based Hypermedia for Individual Learning and Collaborative Knowledge Building", Chapter II, in Eshaa Alkalifa (ed), Cognitively Informed Systems: Utilizing Practical Approaches to Enrich Information Presentation and Transfer, Idea Group Publishing, ISBN: 1-59140-842-3 (hard cover) 1-59140-843-1 (soft cover), pp.26-49, January 2006.

CHAVES, E.O.C. Informática : Micro Revelações. Campinas : Cartgraf Editora e People Computação,1985.

CORREIA, N.; CHAMBEL, T. Integração Multimedia em Meios e Ambientes Aumentados nos Contextos Educativos e Culturais. Arte e Ciência. 2004.

CORREIA, Nuno; CHAMBEL, Teresa. "Active Video Watching Using Annotation", In Proceedings of ACM Multimedia'99, Orlando, Florida, USA, November 1999.

DAKSS, Jonathan. HyperActive: An Automated Tool for Creating Hyperlinked Video. Submitted To The Program in Media Arts and Sciences, School of Architecture and Planning On August 6, 1999. In Partial Fulfillment Of The Requirements For The Degree Of Master Of Science in Media Technology.

DAVIS, H. C.; HALL, W.; HEATH, I.; HILL, G. J.; WILKINS, R. J. Towards an integrated environment with open hypermedia systems. ACM Conference on Hypertext (Hypertext'92). Proceedings....Milan, Italy, December 1992.

FALKEMBACH, G.A.M. Concepção e Desenvolvimento de Material Educativo Digital. V Ciclo de Palestras Novas Tecnologias na Educação. RENOTE. V. 3 N $^{\circ} 1$, Maio, 2005.

GIBBS, G. Improving the quality of student learning. 1992. Technical and Educational Services.

GUIMARÃES, N.; CHAMBEL, T. (2000). "From cognitive maps to hypervideo: Supporting flexible and rich learner-centered environments." Interactive Multimedia Electronic Journal of Computer-Enhanced Learning 2(3). 
MARSHALL C. C. Toward an ecology of hypertext annotation. HYPERTEXT '98: Proceedings of the ninth ACM conference on Hypertext and hypermedia. Maio de 1998.

MORAN, J.M. O vídeo em sala de aula. Comunicacão e Educação, n.2, p. 27-35, 1995.

MOREIRA, M.A. Aprendizagem significativa. Brasília: Ed. da UnB, 1998.

NOVAK, J.D., A Theory of Education, Ithaca, N.Y.: Cornell Uni. Press, 1977.

PATROCINIO, J. M. Hipervídeo. 2006. Acesso: http://www.fafich.ufmg.br/ espcom/ revista/numero1/ArtigoJanainaPatrocinio.html. Em: 17/08/2006.

PETKOVIC, D.E.; LANK, E.; RAMIREZ, F.A.; Raghavendra, S.; CHEN, F.; PEKINER, C.; FREGOSO, A.; MARQUEZ, A. Asynchronous Multimedia Annotations for Web-Base Collaboration in Biology Education. 2005. Computer Science Department* Biology Department. San Francisco State University, 1600. Holloway Avenue, San Francisco, CA, 94132.

PIMENTEL, A.R. et al, "Medidas cognitivas para o ensino de conceitos visuais com Sistemas Tutoriais Inteligentes." - SBIE'97, São José dos Campos - SP, Novembro1997.

PRIMO, A.; RECUERO, R.. Co-links: Proposta de uma nova tecnologia para a escrita coletiva de links multidirecionais. Revista Fronteiras - estudos midiáticos VI(1):91-113, janeiro/junho 2004 @ 2004 by Unisinos.

SAWHNEY, N., BALCOM, D., SMITH, I. HyperCafe: narrative and aesthetic properties of hypervideo. Proceedings of the the Seventh ACM Conference on Hypertext and Hypermedia. Bethesda, Maryland, USA. 1996.

SHIPMAN, F.; GIRGENSOHN, A.; WILCOX L. Hypervideo Expression: Experiences with Hyper-Hitchcock. Conference on Hypertext and Hypermedia. Proceedings of the sixteenth ACM conference on Hypertext and hypermedia. Salzburg, Austria. SESSION: Narratives. Pages: 217 - 226. Year of Publication: 2005 ISBN:159593-168-6.

STOTTS, D., SMITH, J., WILLIAMS, L. Hypervideo Support for Distributed Extreme Programming. Technical Report TR02-009. Março de 2002. Disponível em: rockfish.cs.unc.edu/pubs/TR02-009.pdf. Acessado em maio de 2008.

TAROUCO, L.M.F. Aplicação de teorias cognitivas ao projeto de objetos de aprendizagem. VII Ciclo de Palestras. Revista RENOTE. V. 4 N $^{\circ}$ 2, Dezembro, 2006.

THÜRING, M.; HANNEMANN,J.; HAAKE, J."Hypermedia and Cognition: Designing for Comprehension”, Communications of the ACM, 38(8), 57-66, 1995.

TIELLET, C.A.B. Programa de Informática para o ensino interativo em Cirurgia Veterinária. 2001. 94f. Dissertação (Mestrado em Engenharia de Produção - Tecnologia da Informação) - Universidade Federal de Santa Maria, Santa Maria, 2001. 
TIELLET, C.A.B. Projeto Hipermídia no Ensino: Produção de Objetos de Aprendizagem Hipermídia Através de Aprendizagem Colaborativa. In: International Association for the Scientific Knowledge - Intertic 2007, 2007, Porto. Livro de Actas da Conferência Ibero-Americana. Porto, 2007. p. 234-244. 